\title{
STUDYING OSTEOARTHRITIS PATHOGENESIS IN MICE
}

Running Title: Osteoarthritis in Mice

Andrew Blease ${ }^{1}$, Patricia Das Neves Borges ${ }^{2}$, Marcia Curtinha ${ }^{4}$, Behzad Javaheri ${ }^{3}$, Isabell S von Loga ${ }^{4}$, Ida Parisi ${ }^{4}$, Jadwiga Zarebska ${ }^{4}$, Andrew Pitsillides ${ }^{3}$, Tonia L. Vincent ${ }^{4}$, Paul K. Potter ${ }^{1 *}$

Contact information

1 Disease Model Discovery, Mammalian Genetics Unit, MRC Harwell Institute, Oxfordshire, OX10 9PD, UK

2 Institute of Physiology and Coimbra Institute for Clinical and Biomedical Research (iCBR)- Faculty of Medicine - University of Coimbra, Campus III, Subunit 1, Azinhaga de Santa Comba, Celas, 3000548 Coimbra, Portugal

3 Skeletal Biology Group, Comparative Biomedical Sciences, The Royal Veterinary College, Royal College Street, London, NW1 0TU, UK

4 Arthritis Research UK Centre for OA Pathogenesis, Kennedy Institute of Rheumatology, University of Oxford, Roosevelt Drive, Headington, Oxford OX3 7FY, UK

\section{ABSTRACT}

With the increasing availability and complexity of mouse models of disease, either spontaneous or induced, there is a concomitant increase in their use in the analysis of pathogenesis. Among such diseases is osteoarthritis, a debilitating disease with few treatment options. Whilst advances in our understanding of the pathogenesis of osteoarthritis has advanced through clinical investigations and genome wide association studies there is still a large gap in our knowledge, hindering advances in therapy. Patient samples are available ex vivo but these are generally in the very late stages of disease. However, with mice, we are able to induce disease at a defined time and track the progression in vivo and ex vivo, from inception to end stage, to delineate the processes involved in disease development.

Keywords: Osteoarthritis, Histology, Destabilised Medial Meniscus (DMM), $\mu \mathrm{CT}$, Mouse Models

\section{INTRODUCTION}

Osteoarthritis (OA) is a chronic disease affecting the whole joint; Bone, synovium, synovial fluid, tendons, ligaments, and the capsule are all affected. The combined factors of an aging population and increased risk of disease with aging are resulting in a significant clinical burden with few treatment options beyond pain relief and surgery (McAlindon et al, 2014, Ondresik et al, 2017). The key advantage of mouse models is the ability to study disease progression and intervene in this process at different stages through a variety of techniques such as genetic modification, conditional deletion, and -omics studies that are difficult to carry out on patients due to the difficulty in obtaining samples and appropriate controls. It is not possible to predict who will develop OA and as such, it is difficult to track disease progression in patients, particularly as this generally has to be done with non-invasive techniques primarily imaging technologies such as X ray or MRI. Furthermore, samples from patients are almost exclusively from late-stage disease, meaning there is a great deal of bone remodeling and cartilage erosion and therefore it is difficult to tease out the early processes occurring in disease.

The similarities of joint structure and biology, combined with the vast array of genetic, proteomic, environmental and phenotyping techniques that are currently available, make the mouse an good model organism with which to study this disease. There are spontaneous models of OA, the most commonly employed of these are the Dunkin Hartley Guinea pig (Bendele, Whilte, \& Hulman, 1989) 
and the STR/ort mouse line (Staines, Poulet, Wentworth \& Pitsillides, 2017). There are also chemically induced models but here we focus on the mechanical induction of OA that provides a defined time course and recapitulates many important features of human disease (Fang \& Beier, 2014; Lorenz \& Grassel, 2014).

This review will describe destabilization of the medial meniscus (DMM) surgery (Glasson, Blanchet, \& Morris, 2007) (Vincent, Williams, Maciewicz, Silman, Garside, 2012a), frequently used for studying OA, and a non-surgical method of inducing mechanical damage. Histology provides a detailed method of analyzing disease through the direct visualization of changes in bone and cartilage to assess progression, in both patients and mice but detailed 3D imaging of joints through $\mu \mathrm{CT}$ is also increasingly employed as a non-invasive and/or highly sensitive technique to track disease and we describe some applications of this technique to OA.

\section{BASIC PROTOCOL 1}

\section{SURGICAL INDUCTION OF OSTEOARTHRITIS}

Here we describe the surgical induction of OA through destabilization of the knee joint. The medial meniscotibial ligament is cut to induce the disease. The surgery is straightforward and reproducible when carefully variables are carefully controlled. There is also a wealth of published data describing methodologies and scoring metrics that exhibit subtle changes across different groups although are largely based around the OARSI histopathology score(Aigner et al., 2010; Glasson, Chambers, van den Berg, \& Little, 2010). Disease severity is both gender and strain dependent and this needs to be controlled in all experiments (Glasson, 2007; Glasson, Blanchet, \& Morris, 2007; Ma et al., 2007). Cage occupancy affects mouse behaviour and this also needs to be controlled, typically ensuring greater or equal to 4 animals per cage.

\section{Materials}

\section{Equipment:}

Laminar Air Flow (LAF) cabinet ( \#ACB-6A1, ESCO Life Sciences) Wahl Battery Pet Trimmer Kit (Wahl)

Small animal anaesthesia workstation including mask and box (Stinger streamline \#0873, AAS)

Oxygen concentrator (Nuvo Lite Mark 5 \#935, GCE Medline) (or oxygen cylinder) Dissecting microscope(Nikon SMZ800)

Cold light source (Photonic \#PL2000

Surgical needle holders (World Precision Instruments Ltd \#500223)

Surgical \#5 Dumont jeweler's forceps (World Precision Instruments Ltd \# 500342)

Surgical forceps (World Precision Instruments Ltd \#15915)

Scissors (World Precision Instruments Ltd \# 503222)

Recovery chamber/Hot box (Thermacage Datesand)

Perspex surgery plate ((Made in house, sheet of Perspex $(18 \mathrm{~cm} \times 14 \mathrm{~cm}))$

Sterile Cotton buds/surgical spears

Trigene(or other suitable disinfectant)

lodine/Chlorhexidene (Hydrex Clear, Medisave UK -3030470)

Isoflurane (Zoetis-100\% Isoflurane)

Sterile surgical gloves (Gammex Latex Powder Free Sterile Surgical Procedure Gloves -Ansell

330048065)

Sterile swabs (Medisave UK -cs1850)

15 blade disposable scalpel (Swann Morton \#0505)

$15^{\circ} 3 \mathrm{~mm}$ Micro Surgical Knife (stab knife)- (World Precision Instruments Ltd \# 501731)

Vicryl 6-0 (0.7 Ph.EuR) round bodied needle suture kit (Ethicon W1601T) 
Ethilon 5-0 (1 Ph.EuR) reverse cutting needle suture kit (Ethicon W9575)

Autoclave bag

Autoclave tape

Micropore tape

$1 \mathrm{ml}$ syringe (BD Plastipak, 303172 Medisave UK)

26 gauge sterile needles (BD Microlance 3, ND400 Medisave UK)

Sterile water for injection (WIA010, Medisave UK)

Vetergesic (Ceva Animal health $-0.3 \mathrm{mg} / \mathrm{ml}$ )

Torbugesic (Zoetis $10 \mathrm{mg} / \mathrm{ml}$ )

Viscotears (Novartis \# 2082642)

The following items should be autoclaved, prior to use, in sufficient quantities to complete the required numbers of surgeries; needle holders, scissors, jeweler's forceps, surgical forceps, cotton buds, gauze swaps, and surgical drapes.

\section{Preparation of anaesthesia equipment and analgesics}

1. Dilute $1 \mathrm{ml} 0.3 \mathrm{mg} / \mathrm{ml}$ Vetergesic with $11 \mathrm{ml}$ of sterile water for injection to give a final concentration of $0.025 \mathrm{mg} / \mathrm{ml}$

2. Dilute $0.1 \mathrm{ml} 10 \mathrm{mg} / \mathrm{ml}$ Torbugesic with $3.9 \mathrm{ml}$ Sterile water to give a final concentration of $0.25 \mathrm{mg} / \mathrm{ml}$

3. Turn on Oxygen cylinder or oxygen concentrator (allow 5 minutes for the correct concentration of oxygen, if using the concentrator).

4. Ensure the flow of oxygen is 21 per minute

\section{Surgery}

5. Clean surgical area including microscope

6. Open autoclave bag and lay out sterile drape

7. Open autoclave bag and tip surgical kit onto drape

8. Open microsurgical knife and tip onto drape

9. Open disposable scalpel and tip onto drape

10. Open Vicryl suture kit and tip onto drape

11. Open Ethilon suture kit and tip onto drape

12. Identify appropriate mouse, weigh and place in anaesthetic box, and turn on Isoflurane to induce anaesthesia

Note: The suggested concentration for inducing anaesthesia in rodents is 3-5\% (Table 1), typically we found $3 \%$ good for induction

13. When the mouse is sedated sufficiently (characterized by a lack of response to sound and touch stimuli such as as clicking fingers, brushing whiskers and an absence of a pedal reflex), transfer to preparation area, and place on anaesthetic mask.

14. Shave the anterior right knee.

15. Using tape, collect loose hair and discard.

16. Administer $0.1 \mathrm{ml}$ Vetergestic dilution $(0.0025 \mathrm{mg} @ 0.025 \mathrm{mg} / \mathrm{ml}$ ) via subcutaneous injection

17. Administer $0.1 \mathrm{ml}$ Tourbugesic dilution $(0.025 \mathrm{mg} @ 0.25 \mathrm{mg} / \mathrm{ml}$ ) via subcutaneous injection 
Note: The suggested doses are Vetergesic- $0.05-0.1 \mathrm{mg} / \mathrm{kg}$ and Torbugesic- $1-5 \mathrm{mg} / \mathrm{kg}$, we use $0.1 \mathrm{mg} / \mathrm{kg}$ (assuming mouse is $25 \mathrm{~g}$ ) and $1 \mathrm{mg} / \mathrm{kg}$ (assuming mouse is $25 \mathrm{~g}$ ) respectively.

18. Swab shaved area with lodine/Chlorhexidine solution.

19. Administer viscotears to prevent eyes drying out.

20. Transfer mouse to surgery plate.

21. Position mouse on its back with nose in mask.

22. Turn on isoflurane flow to the mask to maintain appropriate level of anaesthesia and reduce concentration for maintance, check pedal reflex is absent

Note: The suggested concentration for maintaining anaesthesia in rodents is .25-3\% depending on the size of the animal (Table 1), typically we found $0.5 \%$ good for maintance

23. Cover mouse with small sterile drape with right leg through the hole.

24. Remove gloves and put on sterile surgical gloves following correct aseptic technique.

25. Transfer surgery plate to microscope.

26. Reposition surgery tray to enable the right knee to be observed through the microscope.

27. Check that the mouse still has no corneal or pedal reflex before starting surgery and check periodically throughout the surgery to ensure the mouse is still anesthetized.

28. Make initial incision medially to the knee with the 15 blade, approximately $8-12 \mathrm{~mm}$ long, exposing the white parapatellar ligament.

29. Make the secondary incision medially to the white parapatellar ligament.

30. Staunch any bleeding with a sterile cotton bud/surgical spear.

31. Open the incision with the jeweler's forceps

Note: Fine jewelers forceps, can get damaged very easily, use tip guards to protect the points when not in use

32. Identify the medial menisco-tibial ligament (MMTL), using the jeweler's forceps, blunt dissecting the fat pads as necessary.

33. Insert the micro surgical knife into the incision below where the MMTL was identified, with the blade facing up.

34. Apply gentle but steady pressure upwards, until you feel the ligament snap.

35. Staunch any bleeding with a sterile cotton bud/surgical spear.

36. Identify the cut end of the MMTL, the cut should have been fairly close to the tibia, and the loose MMTL running from the MM should be long enough to just protrude from the incision (Figure 1).

37. Further blunt dissection of the fat pads may be necessary to identify the cut end of the MMTL.

38. If the cut end is not visible, the MMTL may not have been completely severed.

39. In which case re-insert the jeweler's forceps where the MMTL was initially identified and see if you can locate the intact (or partially intact) ligament.

40. If this is the case return to step 32 .

41. When the MMTL is definitely severed, close the capsule with a surgeon's knot using Vicryl suture material, 1 suture should be sufficient. 
42. Then close the dermal layer, again using a surgeon's knot with Ethilon suture material, depending on the length of the incision, 2-3 sutures should be sufficient. Ensure the loose ends are cut fairly close to the skin to prevent the animal pulling the sutures.

43. Refresh Viscotears and place mouse in Hotbox or recovery chamber.

44. Repeat from step 12 with all mice.

Mice may have to be individually housed immediately post-surgery if using a recovery chamber, however should be returned to their original home cage once the animal are weight bearing.

Optimal time for each mouse undergoing anaesthesia and procedure should be under 15 minutes. Mice recover from anaesthesia within 5-10 minutes after isoflurane is stopped. There should be no change in locomotion and the mice should be weight bearing equally on the hind limbs.

45. When all mice are recovered and weight bearing return to their home cage in the ward. Our Local and UK requirements require the following information to be recorded on a card associated with the cage; Drugs administered, DMM Surgery, anesthesia code (ABG), your specific licensing number or code, project licence number, protocol licence number, and date.

Note: Please ensure your local and national regulations and guidelines are adhered to throughout all procedures described here.

The amount of time between surgery and culling of the animals can vary, the severity of the disease induced increases with the length of time. The animals are normally culled between 6 and 10 weeks post-surgery, depending on the specifics of the experiment. Once the animal has been culled the hind legs are fixed in $10 \%$ buffered formalin.

\section{ALTERNATE PROTOCOL 1}

\section{Mechanical Induction of Osteoarthritis}

It is known that osteoarthritis degeneration is at least partly attributable to joint loading (i.e. weight bearing across the joint); this is exploited in murine models where abnormal load distributions are imposed by in vivo joint destabilisation to induce OA-like lesions (Little \& Hunter, 2013; Vincent et al., 2012). These models are, however, complicated by surgery, limited by the intransient mechanical joint insult which they impose and hampered by their inability to distinguish between lesion induction and OA progression. We have developed a non-surgical loading model that uniquely allows control and adjustability of the specific mechanical factors applied (e.g. magnitude, number of cycles, hold time, etc.), and evaluation of ensuing impact on articular cartilage integrity in intact joints of (De Souza et al., 2005; Poulet, Hamilton, Shefelbine, \& Pitsillides, 2011) (4,5). Using this model, we find that a few brief cyclical loading episodes, induce overt, load magnitude-dependent OA-like lesions reproducibly in only the lateral femur compartment of mouse knee joints (C57/BL6J and CBA strain). We also find that the number of consecutive applied loading episodes predicts the severity of these OA-like lesions, which worsen in the articular cartilage with solely habitual, subsequent use (De Souza et al., 2005; Poulet et al., 2011, Christiansen et al.,, 2015); the first murine model where articular cartilage deteriorates even after cessation of a transient, controlled and adjustable loading force.

\section{Materials}

1. Material testing unit (e.g. Dartec $\mathrm{HC10}$ servohydraulic testing machine (Fig. 1; Zwick Roell Group, UK)].

2. Anaesthetic agents, if inhalable anaesthesia is used, administer in conjunction with oxygen (e.g. $2-4 \%$ isoflurane) 


\section{Application of loading to mouse knee}

To apply non-invasive axial loading to tibia, various material testing units including Dartec (Zwick/Roell, Germany), EnduraTEC (BOSE Corp, US), Instron Lt (US) and Lloyd Instruments (AMTEK Inc, UK) could be used. These units allow application of a defined load to murine knee without surgical intervention allowing side-to-side comparison between loaded and non-loaded joints. The following procedure is specific to the Dartec HC10 loading device:

1. Before turning on the Dartec machine, screw in the lower cup to the load cell. Load cell is sensitive; to avoid damage do not apply pressure. This cup will protrude through a stage that the anaesthetized mouse will rest on during the procedure. Ensure the stage does not pressure or touch the load cell or the lower cup.

2. Turn on the Dartec machine and associated computer.

3. Open 'Workshop 96' software and select 'Toolkit 96'.

4. On the top toolbar, click on 'Pump Start' to start the hydraulic pump and select 'Main Pressure' to apply hydraulic pressure to the actuator.

5. To prevent damage to the load cell during loading, adjust the 'Offset Number' to a value below the capacity of the load cell. To achieve this, Open the 'Offset/Gains' window and zero the load cell with no sample mounted.

6. To set up desired loading protocol that induces OA, open "Cycle Generator" and then select Waveform'. In this window select "Trapezoid" wave type and 40 for the "Number of Cycles". Enter compressive load of $-2 \mathrm{~N}$ for the low (pre-load) and -9N for high magnitudes of load in 'Level $\mathrm{A}$ and $B^{\prime}$, respectively. Enter 9.9s in "Hold Time A" and 0.05s in "Hold Time B" for the time to be spent at Level A and B. Enter 0.025 for "Fall Time A" and "Fall Time B". Other variables such as 'Amplitude, Mean, Fall Rate and Rise Rate' will be calculated automatically. Click on 'Send', 'Read' to save.

7. To display feedback, select "Tools" and choose "Status". Under 'Settings->Feedback Channel' select Actuator 1; "Clone" this window to display upper and lower loads. Assign one window to "Upper Peak" (the smallest recorded peak value) by selecting "Monitor" and then "Upper Peak". Use the other window to display 'Lower Peak' (the maximum recorded peak value). For both upper and lower peaks select 10 seconds for "Rest Interval". Loading parameters are now set.

8. To prevent damage to the load cell and to ensure accuracy between required and actual load applied to the bone, set the error trips to terminate loading if limits are breached. Under 'Tools>Limits" set the error trips to stop the machine. Error trips detect a difference between the user defined load and the actual load applied. To program error trips, under "Tools->Limits", select Mode-> i.e. Load Cell and 'Lower Limit' - enter in $1 \mathrm{~N}$ over the load cell capacity (i.e. $21 \mathrm{~N}$ ), and choose an 'Action' if the defined limits are exceeded (e.g. Stop Generator).

9. Proceed to inhalant anaesthesia using isoflurane (4\%).

10. To apply load, position the mouse's right ankle in the lower cup and bend the knee so the tibia is vertical (Figure 2).

11. Use the 'Manual Control' window to move the actuator down (- = slow, -- = fast) until a force of approximately $-2 \mathrm{~N}$ (pre-load) is applied to the knee.

12. When the knee is between the upper and lower cups [*Author: what should be the correct placement?], click on 'Start' to begin loading. The mode will change from "Set Up" to "Load Cell". 
13. To visually monitor the progress of loading check the 'Cycle Generator' window, which indicates the number of cycles that are already applied. "Feedback" window shows the actual load that are being applied to the bone.

14. At the end of loading cycle, click on the 'Global Setup' in the top right-hand corner so that the actuator will revert to Set Up mode, the 'Manual Control' window will now display ' $m m$ ', not ' $\mathrm{N}$ '. This will prevent damage to the load cell.

15. To free the mouse knee between the upper and lower cups, use the 'Manual Control' panel to move the actuator up $(+=$ slow,$++=$ fast). Carefully remove mouse and return to cage.

16. Record weight and monitor mouse until fully recovered. The mouse will limp initially post loading.

17. At the end of the experiment, turn off pump using 'Pump Stop' button and carefully remove upper and lower loading cups. Close the software and turn off the machine.

\section{BASIC PROTOCOL 2 - SAMPLE PREPARATION FOR HISTOLOGY}

Histology is still widely used as the gold standard for the evaluation of murine osteoarthritis (OA) and in the past decade has allowed the study of several transgenic mice (Reginato et al., 2002)(Vincent, Williams, Maciewicz, Silman, Garside, 2012b). For instance, the deletion of active ADAMTS5 (Glasson et al., 2005) elucidated the role of degradative enzymes in the progression of the disease.

Histological analysis of murine knee joints has represented a highly valuable tool for a better understanding of the human pathology and giving insights into the molecular basis of this complex and multifactorial disease (Smith et al., 2007; Reginato et al., 2002)(Little \& Hunter, 2013). Mouse knee joints are an important model system to study human OA due to their anatomical similarities to other mammals. However, based on the small size of the specimen macroscopic evaluation of the disease progression is not routinely performed. Microscopic analysis of tissue sections can give an overall view of the entire joint and allow OA disease assessment as explained in more details in Histology - Cartilage Damage Scoring System below.

The semi-quantitative scoring system of mouse cartilage degeneration we describe in this section, is highly reproducible, relatively easy to apply and learn and it has become a common and wellestablished methodology in the OA research field. Other non-cartilage readouts of disease including osteophyte size and maturity, and synovitis are often applied by groups using a 0-3 point scale (Little et al., 2009).

\section{Materials}

$10 \%$ v/v Neutral Buffered formalin (Sigma, HT501128)

Tissue cassettes (Tissue-Tek, \#4170, Sakura)

$20 \%$ (v/v) Formic Acid (Fisher Chemicals F/1850/PB17), 5\% Formalin in distilled water (Sigma, 155512-

1L-R19)

0.5M Ethylenediaminetetraacetic acid (EDTA, 20302.260, VWR) pH 7.4 in distilled water

Tissue processor (Tissue-Tek VIP, Sakura)

$70 \%$ (v/v) Ethanol (Hayman, 200-578-6)

$100 \%$ (v/v) Ethanol (Hayman, 200-578-6)

Xylene (Fisher Chemicals, X10200121)

Molten Paraffin (Cell Path, GCA-0400-00A)

Forceps (VWR 232-00460)

Embedding metallic moulds (Sakura \#4121)

Embedding Centre (Tissue-Tek TEC, Sakura)

Microtome (Leica, RM2235) 
Blades (N35, Cell Path, 02.075.00.006)

Water Bath (Paraffin laboratory water bath Sakura Finetek Europe)

Adhesive microscopic glass slides (Superfrost Plus, 631-0108, VWR)

0.1\% Safranin O dye (Electron Microscopy Sciences, \#20800) 0.5 M acetic acid (VWR, \#20104.334) in distilled water

$0.1 \%$ Fastgreen dye (Sigma, F7252) in distilled water

Harris Haematoxylin (Leica 3801560BDL)

Acid Alcohol: 70\% ethanol (Hayman, 200-578-6) and 1\% hydrochloric acid (Fisher Chemicals, $\# 11150 / \mathrm{PB} / 7$ )

Blueing solution: $0.2 \%$ ammonium hydroxide (VWR, 87766.290) in distilled water, $\mathrm{pH} 10.0$

$1 \%$ Acetic Acid (Sigma \#537020) in distilled water

Glass Coverslips (24x50mm, VWR, ECN 631-1574)

DPX mounting media (Sigma, 06522)

Bright field microscope (Olympus BX51)

Image acquisition software (DP controller, DP Manager, Olympus)

\section{Sample Fixation}

All invasive procedures were approved by the UK Home Office and followed institutional ethical and procedural guidelines.

The mice were euthanized using the inhalant anaesthetic CO2. A secondary physical method of euthanasia such as cervical dislocation was used to ensure death.

Joints are carefully dissected with skin and excessive muscles removal (Figure 3 ). The mouse knees are fixed in $10 \%$ neutral buffered formalin ensuring during this time that the knee is maintained fully extended i.e. straight. Some groups prefer to fix joint in a semi-flexed position as mice weight bear on a bent knee and loaded areas on the tibia and femur are more likely to be directly contacting one another. Tissue may remain in formalin for longer periods until processing if only histological assessment of cartilage damage is required. However, over-fixation (more than 24 hours) must be avoided if immunohistochemistry, immunofluorescence and in-situ hybridization are required together with histological stains as it may be detrimental for the antigene detection. See below for a detailed protocol.

1. The mouse knees are fixed in a volume of $10 \%$ neutral buffered formalin at least 4 times larger than the specimen size for 24 hours.

2. If tissue processing cannot be performed straight after 24 hours fixation, the samples can be stored in $70 \%$ Ethanol for short period.

\section{Decalcification of mouse knees}

$20 \%$ Formic Acid is a fast decalcification method, however the presence of formalin within the solution may cause antigen masking and thus limitations in immunodetection techniques. Therefore, when immunohistochemistry techniques are to be included in the histological analysis, a gentler decalcification method, such as 0.5 M EDTA is preferred. See detailed protocol below.

1. Following fixation, the samples are placed into tissue cassettes.

2. Tissue cassettes should be indelibly labelled with a unique sample identifier. 
3. The samples are then immersed in sufficient volume of $20 \%$ Formic Acid decalcification solution for one week at room temperature (replacement with fresh solution is not necessary) or in 0.5 M EDTA for 2 weeks on a plate shaker and solution is changed every two days.

\section{Tissue processing in paraffin blocks}

The fully decalcified mouse knees can be processed for histological analysis. The samples can be frozen and cryosectioned but for this purpose paraffin embedding is preferred because of the good tissue morphology preservation and cost effectiveness without need of specialised instrumentation.

The tissue cassettes are then transferred to a tissue processor for standard overnight tissue processing. Serial steps of dehydration in $100 \%$ Ethanol and clearing in Xylene are performed followed by infiltration of molten wax into the tissue. See below for a detailed protocol.

1. 1 hour and 30 mins in Formal saline at $40{ }^{\circ} \mathrm{C}$

2. 1 hour and 30 mins in $70 \%$ Ethanol at $40{ }^{\circ} \mathrm{C}$

3. 5 changes of $100 \%$ Ethanol for 1 hour and 30 minutes each at $40{ }^{\circ} \mathrm{C}$

4. 3 changes of Xylene for 1 hour and 30 mins at $40^{\circ} \mathrm{C}$

5. 4 changes of paraffin for 1 hour and 30 mins at $63{ }^{\circ} \mathrm{C}$

If an automatized tissue processing system is not available, manual tissue processing can be performed as followed:

6. The samples are placed overnight in $70 \%$ Ethanol

7. Then they are transferred in $90 \%$ Ethanol for 2 hours

8. Samples are moved in $100 \%$ Ethanol for 2 hours

9. Two changes of Xylene two hours each are performed

10. The specimens are incubated in melted paraffin in the oven at $60{ }^{\circ} \mathrm{C}$ overnight

\section{Tissue embedding, sectioning and staining}

The orientation of the sample is crucial at this stage as coronal embedding allows for simultaneous assessment of the medial and lateral tibio-femoral joints. The evaluation of the entire joint is important as the pathology in mouse models of OA or in genetically modified animals cannot be expected to occur only in the medial compartment of the joint.

11. Fill a mould of appropriate depth and size with molten paraffin wax and transfer the sample from the tissue cassette to the metallic mould on the hot plate, with heated forceps.

12. To embed the joints coronally the patella tendon should be positioned at the bottom of the metallic mould along its long axis (Figure 3, Figure 4, Video 1).

13. After orienting the sample, move the mould to the cold plate carefully to avoid changes in the position of the sample.

14. Cool paraffin blocks enough before sectioning.

Place the paraffin block in the microtome block holder parallel to the blade (Figure 5) in order to avoid folding of the articular cartilage during sectioning, which can prevent the disease scoring (Figure 6).

15. Trim the block until menisci on the medial and lateral compartments of the joint appear distinct. This can be monitored in unstained sections. 
Note: When trimming the block care is needed to see if the orientation is correct (Figure 7). If it is not, the sample can be rotated using the block adjustment screws in the microtome. However if the orientation is sagittal rather than coronal the block must be melted and the sample reembedded.

16. Start collecting $4 \mu \mathrm{m}$ tissue sections.

Note: Keep the block cool and hydrated as it facilitates the sectioning process.

17. 15 levels should be obtained for each block, at $80 \mu \mathrm{m}$ intervals, allowing the examination of the articular cartilage throughout the joint.

18. Two sections can be placed on each slide to ensure scoring of the four joint compartments and avoid the problems caused by histologic artefacts eventually occurring in one section.

19. Float sections onto adhesive glass slides

20. Bake slides overnight at $60^{\circ} \mathrm{C}$ before staining

\section{Staining sections}

An automated stainer should be used in order to keep the staining consistent. The following protocol describes the steps to achieve a satisfactory Safranin-O/Fast-Green staining of knee joint sections (Figure 7).

\section{Safranin-O/Fast Green staining:}

21. Dewax sections in 2 changes of Xylene 5 minutes each

22. Hydrate sections ( 3 changes of $100 \%$ ethanol one minute each followed by running tap water for 20 seconds)

23. Place sections in Harris haematoxilyn for 30 seconds

24. Wash sections in running water for 1 minute

25. Differentiate with acid alcohol for 20 seconds

26. Wash sections in running water for 1 minute

27. Place the sections in ammonia water for 1 minute

28. Stain with Fast Green for 6 minutes

29. Rinse with $1 \%$ acetic acid for 15 seconds

30. Wash sections in running water for 1 minute

31. Stain with Safranin-O for 2 minutes

32. Wash sections in running water for 1 minute

33. Dehydrate ( $3 \times 1$ minute $100 \%$ ethanol), clear ( $2 \times 1$ minute xylene) and mount with DPX

Imaging and histological assessment of cartilage damage

34. Take images of both joint compartments (lateral and medial) at 10x magnification with an image acquisition software (Figure 8, B and C). 
35. Repeat this process for at least 9 of the 15 levels, in order to have a representative view of the articular cartilage throughout the entire joint and therefore a more accurate disease scoring.

36. Score the images using an appropriate scoring system (see below).

\section{BASIC PROTOCOL 3 - MICRO-COMPUTED TOMOGRAPHY OF BONE IN MICE}

\section{Introduction}

In the recent years, micro-computed tomography $(\mu \mathrm{CT})$ has become the gold standard non-invasive imaging modality for bone assessment in rodents owing to the excellent spatial resolution combined with 3D capability. Harmless radiation doses and increasingly faster scans not only allow the crosssectional study of harvested samples (Das Neves Borges, Vincent, \& Marenzana, 2017), but also the longitudinal assessment of live animals (Laperre et al., 2011).

$\mu C T$ results in the 3D visualisation of bone microstructure with a fine level of detail. Figure $9 A$ shows different views of a 3D reconstruction of a mouse knee joint and representative 2D slices (Figure 9B), enhancing the microarchitectural features observed in cortical and trabecular bone. 2D and 3D morphometric quantifications can be obtained to assess structural properties. In cortical bone, these include, but are not limited to, average thickness, total volume and area, endosteal and periosteal perimeters, as well as porosity and bone mineral density. On trabecular bone, the thickness, number, separation, and connectivity of trabeculae, as well as bone volume fraction and bone mineral density (Mary L Bouxsein et al., 2010). Some of these measures are more relevant to osteoporosis rather than osteoarthritis but increased density is indicative of increased mechanical load passing through the medial side of the joint. Other measures that are disease specific include subchondral bone sclerosis and osteophyte size measurement(Neves Borges, Vincent, \& Marenzana, 2017).

The following protocol describes the procedure to image bone specimens that have been freshly dissected and fixed in 10\% buffered formalin (Sigma, HT501128) for 24 hours immediately after dissection and subsequently preserved in 70\% (v/v) ethanol (Hayman, 200-578-6). Most of the adjacent soft tissue should be dissected prior to imaging but minimal sample preparation is required for the procedure.

\section{Materials}

Tweezers (VWR, 232-0090)

Corning ${ }^{\circledR} 15 \mathrm{~mL}$ centrifuge tube (Sigma, CLS430791)

1x phosphate buffered saline (Sigma, P4417), pH 7.4

$7.5 \mathrm{~mL}$ graduated transfer pipette (Thermo Scientific ${ }^{\mathrm{TM}}$, 15251799) Non-PVC cling film (similar to Sainsbury's cling film, 7650540)

$5 \mathrm{~mL}$ plastic sample tube (similar to Thermo Scientific ${ }^{\text {TM }}$, 343923) ]CT scanner (such as Bruker, Skyscan 1172)

\section{Sample Preparation}

1. Use the tweezers to transfer the sample from $70 \%(\mathrm{v} / \mathrm{v})$ ethanol into the $15 \mathrm{~mL}$ centrifuge tube and fill it with phosphate buffered saline (PBS) using the graduated transfer pipette. Allow tissue re-hydration for 30 minutes at room temperature.

2. To avoid movement throughout image acquisition, constrain the sample by thoroughly wrapping it in multiple layers of cling film and vertically place it into the sample container half filled with PBS. 
3. Add solution until the specimen is covered in aqueous media and proceed with image acquisition upon defining the volume to be imaged, as well as the resolution and time of exposure desired (for detailed steps on the use of Bruker's software for image acquisition follow the protocols documented on bruker-microct.com/).

4. Upon imaging, carefully remove the layers of cling film and return the specimen to $70 \%$ ethanol using the tweezers.

\section{SUPPORT PROTOCOL 1 - CALIBRATION FOR BONE MINERAL DENSITY (BMD)}

To obtain measurements of bone mineral density (BMD), a linear relationship between the grey level intensity and BMD must be derived using high and low density $\left(0.75\right.$ and $0.25 \mathrm{~g} / \mathrm{cm}^{3}$ of calcium hydroxyapatite, respectively) phantom rods provided by scanner manufacturers. The cross-sectional diameter of such phantoms must match the cross-sectional thickness of mouse long bones and the scanning and reconstruction settings must be identical to the ones used for the specimens. Upon scanning (both rods can be imaged within the same tube if appropriately stacked) and reconstruction, the steps illustrated in Figure 10 should be considered to effectively perform the calibration:

1. By means of an image analysis software, such as ImageJ (US National Institutes of Health, Bethesda, Maryland, USA) (Schneider, Rasband et al. 2012), select a volume of interest (VOI) in the central area of both calibration rods and across at least 100 slices; avoid outer and top surfaces.

2. From the histogram of grey level intensity, obtain the mean grey level intensity within both VOIs (for detailed steps on the use of ImageJ software follow the user's guide available on imagej.nih.gov/ij/docs/guide).

3. Based on the principle that in $\mu \mathrm{CT}$, grey level intensity and $\mathrm{BMD}$ have a linear relationship (Kazakia, Burghardt, Cheung, \& Majumdar, 2008), derive the linear calibration that transforms the grey level intensity of each pixel into the correspondent value of BMD (expressed in $\mathrm{g} / \mathrm{cm}^{3}$ ).

\section{SUPPORT PROTOCOL 2 - CALIBRATION IN HOUNSFIELD UNITS (HU)}

For X-ray attenuation studies, CT numbers, which are measured in Hounsfield units (HU), must be obtained. For this purpose, a calibration should be performed in similar manner as detailed for BMD, but in which air and water are used as radiodensity references (-1000 and $0 \mathrm{HU}$, respectively). Consider the following steps:

1. Scan a sample tube half filled with distilled water in such way that the volume imaged includes both air and water; in this way, only one scan is required for both references. Perform the imaging and reconstruction using the same settings as the specimens.

2. Using an image analysis software, such as ImageJ (Schneider, Rasband et al. 2012), determine the average grey level intensity within a VOI across at least 100 slices in the water and air scans; avoid outer and top surfaces.

3. Considering that water is defined to have $0 \mathrm{HU}$ of radiodensity and air is established to have - 1000 $\mathrm{HU}$, derive the linear calibration that transforms the grey level intensity of each pixel into the correspondent attenuation in $\mathrm{HU}$.

\section{BASIC PROTOCOL 4 - EX VIVO IMAGING OF CARTILAGE}




\section{Introductory paragraph}

The following protocol describes the procedure to image articular cartilage of long bones using phosphotungstic acid (PTA) as contrast agent (Das Neves Borges, Forte, Vincent, Dini, \& Marenzana, 2014). Specimens should be disarticulated and finely dissected, and subsequently fixed in $10 \%$ buffered formalin (Sigma, HT501128) for 24 hours and subsequently preserved in 70\% (v/v) ethanol.

\section{Materials}

$20 \mathrm{~mL}$ of $1 \%$ phosphotungstic acid hydrate (Sigma, P4006) in 70\% ethanol (Hayman, 200-578-6) (w/v) at room temperature and freshly prepared

Tweezers (VWR, 232-0090)

Corning ${ }^{\circledR} 15 \mathrm{~mL}$ centrifuge tube (Sigma, CLS430791)

$7.5 \mathrm{~mL}$ graduated transfer pipette (Thermo Scientific ${ }^{\mathrm{TM}}$, 15251799)

Non-PVC cling film (similar to Sainsbury's cling film, 7650540)

$5 \mathrm{~mL}$ plastic sample tube (similar to Thermo Scientific ${ }^{\mathrm{TM}}, 343923$ )

?CT scanner (Bruker, Skyscan 1172)

\section{Sample Preparation}

1. Use the tweezers to transfer the sample from $70 \%(\mathrm{v} / \mathrm{v})$ ethanol into the centrifuge tube and fill it with PTA solution using the graduated transfer pipet. Allow tissue staining for 8 to 24 hours at room temperature.

2. To avoid movement throughout image acquisition, constrain the sample by thoroughly wrapping it in multiple layers of cling film and vertically place it into the sample tube half filled with $1 \%$ PTA in $70 \%$ ethanol $(w / v) .$.

3. Add solution until the specimen is covered and continue with image acquisition using the same settings applied for conventional $\mu \mathrm{CT}$ imaging of bone.

4. Upon imaging, carefully remove the layers of cling film and wash the specimen in large volumes of $70 \%(\mathrm{v} / \mathrm{v})$ ethanol to remove PTA.

5. Return the specimen to $70 \%(\mathrm{v} / \mathrm{v})$ ethanol using the specimen tweezer.

\section{SUPPORT PROTOCOL 3- WHOLE KNEE JOINT IMAGING}

In circumstances such as histopathology, it is advantageous to preserve the knee joint intact. In this instance, to effectively perform contrast enhanced $\mu \mathrm{CT}$, it is essential to expose the articular surfaces and assure that they are reachable by the contrast agent without compromising the integrity of the joint. Two main challenges are encountered while performing contrast enhanced $\mu \mathrm{CT}$ of the whole joint: firstly, incomplete staining (Figure 11A) and secondly, overlapping among different articular surfaces (Figure 11B). In the first case, the integrity conferred by the capsule causes a shielding that does not allow the contrast agent (PTA in this case) to stain all articular cartilage surface, leading to the observation of 'gaps' where enhancement was not effective (red arrows in Figure 11A); overlaying among the articular surfaces poses difficulties assessing each joint component. To minimise these issues, consider the following procedure for articular cartilage imaging in the knee joint:

1. Thoroughly dissect the soft tissue, open the knee capsule, and remove the patella (menisci and medial and lateral ligaments can also be carefully dissected).

2. Incubate the joint in $1 \%$ PTA in $70 \%$ ethanol $(w / v)$ for 24 hours at room temperature, preferentially in such way that the femur is flexed to allow full staining of the articular surfaces. 
3. Prior to image acquisition, position the tibia vertically and the femur flexed, wrap the joint in multiple layers of cling film and place it into the sample tube half filled with $1 \%$ PTA in $70 \%$ ethanol $(w / v)$.

4. Add solution until the specimen is covered and proceed with image acquisition using the same settings and parameters of split joints.

5. Upon imaging, carefully remove the layers of cling film and wash the specimen in large volumes of $70 \%$ ethanol to remove PTA.

6. Return the specimen to $70 \%$ ethanol using the specimen tweezer.

\section{BASIC PROTOCOL 5 - MURINE PAIN MEASUREMENTS - LINTON INCAPACITANCE TESTER}

There are several ways to measure painful behaviour in mice - we found the most robust as well as clinically and physiologically relevant measure to be static weight distribution measurement. The following protocol describes the procedure of using the Linton Incapacitance Tester to assess spontaneous painful behaviour in mice with murine OA.

\section{Materials}

Linton incapacitance tester (Linton Instrumentation, Incapacitance)

Specimen tweezer (VWR, 232-0107)

A small sturdy piece of paper

1. Bring the animal cages to the testing room with as little disturbance as possible

2. Let them acclimatize in their cages for 15-30 minutes

3. During this time, calibrate the Linton incapacitance tester (Figure 12A) using the $50 \mathrm{~g}$ weight for both platforms. If need be, also double check the tare and ensure it is set to zero between both platforms. During this time, calibrate the Linton incapacitance tester (Figure 12A) using the $50 \mathrm{~g}$ weight for both platforms. The instrument requires little to no maintenance, but if necessary, please consult the manual for further maintenance steps.

4. Set your recording time to 1 second interval (NB: Other groups working with rats use 3 second intervals. We found that due to mice being inherently more active, a 1 second interval is more suitable).

5. One by one, move the animal from the cage into the Linton incapacitance box with as little handling disturbance as possible. Best is to let the animals walk off the experimenter's arm into the box by themselves as to prevent a stress-response.

6. Once in the box, wait until the animal spontaneously turns into the correct position with the forelimbs resting on the curved side of the box

7. Lightly extract the tail using the specimen tweezers - take care as to not disturb or startle the animals by doing so. Lightly hold the tail to ensure the animals stays in the correct position

8. Using the small sturdy sheet of paper, reposition the hindlimbs if necessary, to encourage a symmetric stance (see Figure 12B). 
9. When the animal is resting in the correct symmetrical position, hit "enter" to assess the weight difference

10. Record three back-to-back readings with the animal in the correct position. If the animal moves during this time, wait until it has settled again before recording

11. After obtaining all required readings, return the animal to their cage

12. If necessary, clean the platform and box before starting with the next animal

13. After collection of all readings, calculate an average of all three readings within one animal to calculate the percentage weight difference across both legs.

14. Take the average of all animals within groups to receive a final reading for that group.

\section{COMMENTARY}

\section{Background Information}

We have described two methods of inducing disease in mice, with DMM surgery being the most commonly employed method in current studies. Another method of surgically induced OA is anterior cruciate ligament transection (ACLT) but this is technically more demanding and results in a severe $O A$ (Glasson et al., 2007). In addition to analysing joints through histology or $\mu \mathrm{CT}$, gene expression and proteomic analysis, genetic and drug interventions, and many other techniques have been utilised in OA research. The protocols presented here are all applicable in such studies to induce, study and modulate disease progression. Similarly, spontaneous models can be analysed using the methods described here.

We have also described a robust method of measuring painful behaviour in murine OA. Other frequent measures of pain are evoked measures such as distal mechanical allodynia using von Frey filaments. We found von Frey filaments, albeit in widespread use in the field of inflammatory pain, difficult to apply in osteoarthritic pain. Weight distribution measurement, on the other hand, is a physiologically relevant measure of pain as it intuitively mimics real-life behaviour of offloading a painful joint. Having said this, there is a significant learning curve to achieve reproducible results using this method and care must be taken to avoid bias. Such measures include randomization of treatment (DMM and sham surgery, or placebo and drug), and blinded assessment.

Benchtop imaging systems are composed of a microfocal spot X-ray source that emits a polychromatic beam whose energy band is narrowed by a collimator and filters. Depending on the construction principle, either the specimen is centrally positioned on a rotating stage or a gantry with the source and detector rotates around the subject (M. L. Bouxsein et al., 2010). The first type of design is commonly applied for ex vivo imaging, while the second is intended for in vivo imaging, in which the anaesthetised animal is restrained in a fixed bed throughout image acquisition ( $\mathrm{Li}$, Zhang, Tang, \& Hu, 2008). As the X-ray beam passes through the specimen, emerging photons reach the detector to be collected in a high-resolution charge-coupled device, converted into electrical charges, and digitalized (Schambach, Bag, Schilling, Groden, \& Brockmann, 2010). Several projection images are acquired at fixed angular steps in a total rotation of $180^{\circ}$ or $360^{\circ}$. A 3D dataset is subsequently generated by a reconstruction algorithm often based on the filtered backprojection method originally described by Feldkamp et al. (Feldkamp, Davis, \& Kress, 1984). This algorithm not only processes the projection views to reconstruct the volume, but also compensates for possible imaging artefacts caused by limitations in the experimental setup (Holdsworth \& Thornton, 2002). Additionally, upon imaging live 
animals, gating techniques are applied in the reconstruction to reduce motion artefacts caused by cardiac and respiratory cycles (Brehm, Sawall, Maier, Sauppe, \& Kachelrieß, 2015).

In spite of providing excellent resolution for mineralised tissue imaging, $\mu \mathrm{CT}$ shows poor contrast for soft tissue visualisation owing to its radiolucency (Pauwels, Van Loo, Cornillie, Brabant, \& Van Hoorebeke, 2013). This caveat can, however, be overcome by the use of contrast enhancement agents that improve the visualisation of the tissue of interest (Lusic \& Grinstaff, 2013). The denominated contrast-enhanced $\mu \mathrm{CT}$ comprises a field in substantial development, not only on the screening and application of readily available radiopaque compounds to image different soft tissues, but also on the synthetises of novel, targeted agents. Classically, these compounds have in their composition atoms with high atomic number and possess affinity to the tissue of interest, causing a gradient of attenuation between enhanced and non-enhanced regions (Metscher, 2009). Ex vivo, contrastenhanced [CT involves the incubation of harvested samples in contrast agent prior to imaging, while in vivo it requires the non-hazardous agent to be locally or systemically delivered to the animal. Successful applications of contrast-enhanced $\mu C T$ in the mouse range from 3D visualisation of articular cartilage (Das Neves Borges, Forte, Vincent, Dini, \& Marenzana, 2014; Lakin et al., 2016), vasculature (Gayetskyy et al., 2014; Sutter et al., 2017), kidney (Missbach-Guentner et al., 2018) and heart (Dunmore-Buyze et al., 2014) morphology.

\section{Critical Parameters \\ DMM Surgery}

In the UK surgery is a regulated procedure and therefore can only be carried out by a competent person who holds a Home Office Personal Licence as part of a programme of scientific work as specified in Section E (19b) of a Project Licence within a Licenced Establishment. Ensure you adhere to local regulations and guidelines before commencing invasive techniques such as the surgery described here. Aseptic technique must be followed at all times.

DMM is generally performed on male mice at 10-12 weeks of age, but can also be applied to older mice. It is advisable to practice the technique on a cadaver prior to starting surgery on live animals. It is always worth opening the capsule fully after performing surgery on a cadaver to ensure that your technique is only severing the MMTL, and is doing no other damage. It is standard practice to include sham-operated mice, which undergo the full surgical procedure but without cutting the MMTL, in each cohort undergoing DMM surgery to assess technique. Ideally the mice should be randomly assigned to DMM or sham surgery and the histological scoring also carried out blind (by two individuals) to overcome unconscious bias. It is bad practice to cage cohorts of experimental mice and controls separately; they should be mixed within cages.

It has been reported that the role of sex hormones is critical in the progression of OA in murine DMM. Male mice develop more severe OA than female mice(Ma et al., 2007). When designing the study, this needs to be taken into consideration. It is good practise to use single sex throughout the study, but ideally both sexes would be included in any study.

A pilot study is required to determine your required experimental sample size. The pilot data will generate a standard deviation. A power calculation is then used to determine sample size needed in your study. In line with the $3 \mathrm{Rs}$, it is necessary to determine the number of mice (sample size) which allows you to uncover a significant result for a given effect size with a given degree of confidence. Alternatively, if the sample size is limited, the probability of achieving a significant result with a given effect size can also be determined. A higher power is preferable to ensure results are reproducible, so it is often set at 0.8 or $0.9(80 \%$ or $90 \%)$, but the higher power will require a larger sample size. 
For more information, go to http://www.3rs-

reduction.co.uk/html/6 power and sample size.html. The ARRIVE guidelines are a good starting point when planning an in vivo experiment (Kilkenny, Browne, Cuthill, Emerson, \& Altman, 2010).

The number of mice should be maintained at 4-6 per cage throughout the experiment and across cohorts (or at least consistent across cages and dependent on standard cage size), to control for consistent levels of locomotor activity of the mice. To ensure reproducibility all factors should be consistent between experiments where feasible, and highlighted where they could not be controlled. These factors include; breeding strategies, housing conditions, diet, age, sex and so on.

\section{$\mu C T$ (Bone)- Resolution and field of view}

A spatial resolution up to $10 \mu \mathrm{m} /$ pixel should be used for accurate imaging of trabecular bone in the mouse. As an illustration, Figure 13 shows the same mouse knee joint imaged at an isotropic voxel size of 5 and $10 \mu \mathrm{m}^{3}$; at lower resolution, blurring, particularly on trabeculae is already perceptible, which can decrease the accuracy of structural quantifications. Nonetheless, higher resolutions result in smaller fields of view, limiting both the height and diameter imaged in each scan. In some cases, this caveat can be partially overcome by the control software of the scanner that images larger heights by stitching sequential scans of the object but at the expense of increased scanning times.

\section{$\mu C T$ (Bone) -Image acquisition and reconstruction}

For optimal contrast and minimal beam hardening, the energy of the X-ray source should be set to a voltage ranging between 50 to $70 \mathrm{kV}$ and a current between 160 to $200 \mathrm{~mA}$ (Campbell \& Sophocleous, 2014). Since benchtop systems emit polychromatic beams, artefacts caused by beam hardening can decrease the final image quality. This is a result of the preferential absorption of low energy $X$-rays on surfaces leaving inner regions to appear less dense since remaining high energy X-rays are less absorbed (Brooks \& Di Chio, 1976). To reduced beam hardening during acquisition, an aluminium filter should be used to narrow the energy band of the beam; moreover, artefacts can be removed by applying mathematical corrections during image reconstruction (Postnov, Vinogradov, Van Dyck, Saveliev, \& De Clerck, 2003). Dedicated reconstruction software, such as NRecon from Bruker, allow not only for beam hardening reduction by setting a constant correction factor, but also include ring artefact reduction, misalignment compensation and smoothing. Ring artefacts, which appear as a result of imperfections in the detector and cause concentric rings superimposed on the images, can be minimized by randomly moving the object by a few $\mu \mathrm{m}$ throughout scanning (Raven, 1998); this can be automatically accomplished by the control software during image acquisition. To compensate for possible misalignments, the offset between the $0^{\circ}$ and $180^{\circ}$ (or $360^{\circ}$ ) side projections is calculated by the reconstruction software and an optimal overlay between the two projections is obtained prior to reconstruction. Smoothing, mainly performed by applying a Gaussian filter, is frequently part of the reconstruction process to reduce any noise in the images, but to avoid blurring the images, a filter size not larger than one to two pixels should be applied.

\section{uCT (cartilage) - Resolution}

In naïve mice, articular cartilage has been shown to have a maximum thickness of $80 \mu \mathrm{m}$ (Das Neves Borges et al., 2014), hence for accurate imaging of its morphology, a spatial resolution down to 5 $\mu \mathrm{m} /$ pixel should be used. This is particularly important when applying contrast enhanced $\mu \mathrm{CT}$ to disease models in which damage and progressive loss are observed. Poor resolution does not provide adequate level of detail to detect early to mild morphological changes and may affect subsequent quantitation. Note on Figure 14 the application of contrast-enhanced $\mu$ CT using PTA to tibiae from a surgical model of knee osteoarthritis (destabilisation of the medial meniscus, DMM). At a resolution of $5 \mu \mathrm{m} /$ pixel and upon 24 hours of incubation in contrast agent, is possible not only to visualise the 
morphology of healthy cartilage in controls, but also to observe its gradual damage as disease progresses. On early stages (4 weeks upon osteoarthritis induction), superficial damage is observed as thin cuts that in late stages (12 weeks) cause deeper lesions.

\section{Pain measurements}

During the first time of handling, mice will not voluntarily explore the inside of the box. It is essential that there are several 'dry runs' of the procedure for the animals to get used to being handled and also getting used to sitting still on the Linton incapacitance tester. We recommend at least 3 acclimatization runs spread over several days for the animals to get used to the procedure.

We found the best time to assess the animals on the Linton incapacitance tester to be first thing in the morning before the daily activity of the animal unit starts to disturb the animals. Additionally, testing should be in a quiet environment as to not disturb the animals.

There have been some incidental findings, within our laboratory, that the presence of male assessors overly disturbs the animals leading to a stress response, which is in keeping with a recent discovery showing that olfactory exposure of mice to males leads to masking of painful behaviour (Sorge et al., 2014). Therefore, care should be taken to always keep the same assessor and limit presence of others in the room.

Handling of the animals should be as non-invasive as possible to avoid a further stress response. Additionally, a swift recording in the box is advantageous, as longer time in a limited space will lead to an increased stress response and therefore possible masking of painful behaviour.

\section{Troubleshooting}

\section{DMM Surgery}

Mice can gnaw at the stitches and so the wound can come apart in the 1-3 days post-surgery. It may be therefore necessary to re-stitch. Ensure that the sutures and pulled tight and keep the ends of the suture short. The surgical wound should be monitored daily up to 14 days post DMM for appropriate wound healing, signs of inflammation or infection and suture dispersal. It is not advisable to use antibiotics post-operatively.

\section{$\mu C T$ - Image acquisition}

PTA may lead to non-specific staining of any remaining soft tissue containing collagen, such as tendons and ligaments; consequently, fine image acquisition is recommended. To do so, increase the number of projection images taken at each angular step; while for conventional $\mu \mathrm{CT}$ imaging of bone, 2 frames/projection produce good quality images, 3 frames/projection are recommended for contrastenhanced $\mu \mathrm{CT}$ imaging; longer exposure times also improve the results. Concerning total acquisition time per harvested sample, this will then range from 20 to 25 minutes for conventional $\mu \mathrm{CT}$ and 30 to 40 minutes for contrast-enhanced $\mu \mathrm{CT}$, depending on exposure time, angular step, number of projection images and resolution.

\section{Pain Measurements}

Mice can get agitated if kept in the box for too long. In this case, the animal should be returned to its cage and given time to acclimatize again before restarting the recording.

Most difficulties stem from the animals developing a stress response. Therefore, care should be taken to limit this as much as possible. Avoid performing other testing e.g. other pain assessments or treatments in the animal under test. When it is necessary to do so always stick to the same order of tests. 


\section{Statistical Analyses}

All groups of data are assessed for approximation to the Gaussian distribution using the D'Agostino and Pearson omnibus test of normality (D'Agostino et al 1990). Distributions are considered to be Gaussian if the $P$ value for the null hypothesis is greater than 0.05 . When multiple comparisons between multiple end points are performed, the Bonferroni post hoc test is used to adjust for multiplicity. There are many packages available to facilitate statistical analysis of data including GraphPad Prism. To derive the sample size, power calculations based on previous data in wild type mice 8 weeks post DMM is performed. Initial experiments can be powered to detect a $50 \%$ change in chondropathy score (6 points), based on a standard deviation of 6.7 , with $90 \%$ power and a set at 0.05 . This gives 16 mice ( 8 in each group). Further animals can be added subsequently to increase the power in order to detect a smaller difference between groups. Conditional deletion of genes is often incomplete and produces greater variance. Higher numbers are often required.

\section{Understanding Results}

\section{Histology - Cartilage Damage Scoring System}

Several different scoring systems have been described in the literature to assess $O A$ in the mouse models. These can either limit their evaluation to cartilage damage or also observe bone and synovial changes. Two well described scoring systems were published by Chambers et al. (Chambers, Bayliss, \& Mason, 1997) and Glasson et al. (Glasson, Chambers, Van Den Berg, \& Little, 2010).

The recommended semi-quantitative scoring system is a modification from Glasson et al. (Glasson et al., 2005), in which a score of 0 represents normal cartilage, $0.5=$ loss of proteoglycans staining with intact articular cartilage surface, $1=$ surface fibrillations without loss of cartilage, $2=$ loss of superficial cartilage and surface delamination, with shallow fissures but no frank ulceration, $3=$ ulceration of noncalcified cartilage/cartilage delamination at the tidemark, $5=$ ulceration extending into calcified cartilage but not into subchondral bone, $6=$ ulceration extending into subchondral bone (Figure $15 \mathrm{~A}$ and $\mathrm{B})$.

In this scoring system all four compartments of the joint (medial tibial plateau, medial femoral condyle, lateral tibial plateau and lateral femoral condyle (Figure 15A) within each section are scored separately and the scores are added up to give one score for each section (between 0 and 24). A minimum of 9 levels, for each joint, are scored by 2 independent observers, under blinded conditions.

The histologic summed score (maximum 72) for each joint is calculated from the three highest sections scores to provide an indicator of the severity of cartilage damage. The final score for a given joint is the average of the summed scores from each observer. An example of the histological analysis of the consequences of DMM surgery on wild type mice is shown in Figure 16. With increasing age of wild type mice there is a greater severity of surgery induced disease.

\section{ACKNOWLEDGEMENTS}

PKP and AB were funded by the Medical Research Council, UK, (reference MC U142684172), the Arthritis Research UK Centre for Osteoarthritis Pathogenesis (reference 20205) and the Kennedy Trust for Rheumatology Research. AP and BJ were funded by Arthritis Research UK Grant (reference 205810). TLV, PDNB, JZ, IP, MC and ISVL were funded by the Arthritis Research UK Centre for Osteoarthritis Pathogenesis (reference 20205) and the Kennedy Trust for Rheumatology Research.

\section{LITERATURE CITED}


Aigner, T., Cook, J. L., Gerwin, N., Glasson, S. S., Laverty, S., Little, C. B., et al. (2010). Histopathology atlas of animal model systems - overview of guiding principles. Osteoarthritis and Cartilage / OARS, Osteoarthritis Research Society, 18 Suppl 3, S2-6.

Bendele AM, White SL, Hulman JF (1989). Osteoarthritis in guinea pigs: Histopathologic and scanning electron microscopic features. Laboratory Animal Science, 39, 115-121.

Bouxsein, M. L., Boyd, S. K., Christiansen, B. A., Guldberg, R. E., Jepsen, K. J., \& Müller, R. (2010). Guidelines for assessment of bone microstructure in rodents using micro-computed tomography. J Bone Miner Res., 25(7), 1468-1486.

Bouxsein, M. L., Boyd, S. K., Christiansen, B. A., Guldberg, R. E., Jepsen, K. J., \& Müller, R. (2010). Guidelines for assessment of bone microstructure in rodents using micro-computed tomography. Journal of Bone and Mineral Research, 25(7), 1468-1486.

Brehm, M., Sawall, S., Maier, J., Sauppe, S., \& Kachelrieß, M. (2015). Cardiorespiratory motioncompensated micro-CT image reconstruction using an artifact model-based motion estimation. Medical physics, 42(4), 1948-1958.

Brooks, A. R., \& Di Chio, G. (1976). Beam hardening in X-ray reconstructive tomography. Phys. Med. Biol., 21(3), 390-398.

Campbell, G. M., \& Sophocleous, A. (2014). Quantitative analysis of bone and soft tissue by microcomputed tomography: applications to ex vivo and in vivo studies. BoneKEy reports, 3.

Chambers, M. G., Bayliss, M. T., \& Mason, R. M. (1997). Chondrocyte cytokine and growth factor expression in murine osteoarthritis. Osteoarthritis Cartilage, 5(5), 301-308.

Christiansen, B. A., Guilak, F., Lockwood, K. A., Olson, S. A., Pitsillides, A. A., Sandell, L. J., Silva, M. J., van der Meulen, M. C., and Haudenschild, D. R. (2014). Non-invasive mouse models of posttraumatic osteoarthritis. Osteoarthritis Cartilage 23 (10), 1627-1638.

Das Neves Borges, P., Forte, A. E., Vincent, T. L., Dini, D., \& Marenzana, M. (2014). Rapid, automated imaging of mouse articular cartilage by microCT for early detection of osteoarthritis and finite element modelling of joint mechanics. Osteoarthritis Cartilage, 22(10), 1419-1428. doi:10.1016/j.joca.2014.07.014

Das Neves Borges, P., Vincent, T. L., \& Marenzana, M. (2017). Automated assessment of bone changes in cross-sectional micro-CT studies of murine experimental osteoarthritis. PloS one, 12(3), e0174294. doi:10.1371/journal.pone.0174294

De Souza, R. L., Matsuura, M., Eckstein, F., Rawlinson, S. C., Lanyon, L. E., \& Pitsillides, A. A. (2005). Non-invasive axial loading of mouse tibiae increases cortical bone formation and modifies trabecular organization: a new model to study cortical and cancellous compartments in a single loaded element. Bone, 37(6), 810-818.

Dunmore-Buyze, P. J., Tate, E., Xiang, F. I., Detombe, S. A., Nong, Z., Pickering, J. G., \& Drangova, M. (2014). Three-dimensional imaging of the mouse heart and vasculature using micro-CT and whole-body perfusion of iodine or phosphotungstic acid. Contrast media \& molecular imaging, 9(5), 383-390.

Fang, H., \& Beier, F. (2014). Mouse models of osteoarthritis: modelling risk factors and assessing outcomes. Nat Rev Rheumatol, 10(7), 413-421. doi:10.1038/nrrheum.2014.46

Feldkamp, L. A., Davis, L. C., \& Kress, J. W. (1984). Practical Cone-Beam Algorithm. Journal of the Optical Society of America a-Optics Image Science and Vision, 1(6), 612-619. doi:Doi 10.1364/Josaa.1.000612

Gayetskyy, S., Museyko, O., Käßer, J., Hess, A., Schett, G., \& Engelke, K. (2014). Characterization and quantification of angiogenesis in rheumatoid arthritis in a mouse model using $\mu C T$. $B M C$ musculoskeletal disorders, 15(1), 298.

Glasson, S. S. (2007). In vivo osteoarthritis target validation utilizing genetically-modified mice. Current Drug Targets, 8(2), 367-376.

Glasson, S. S., Askew, R., Sheppard, B., Carito, B., Blanchet, T., Ma, H. L., . . Morris, E. A. (2005). Deletion of active ADAMTS5 prevents cartilage degradation in a murine model of osteoarthritis. Nature, 434(7033), 644-648. 
Glasson, S. S., Blanchet, T. J., \& Morris, E. A. (2007). The surgical destabilization of the medial meniscus (DMM) model of osteoarthritis in the 129/SvEv mouse. Osteoarthritis Cartilage, 15(9), 1061-1069.

Glasson, S. S., Chambers, M. G., Van Den Berg, W. B., \& Little, C. B. (2010). The OARSI histopathology initiative - recommendations for histological assessments of osteoarthritis in the mouse. Osteoarthritis Cartilage, 18 Suppl 3, S17-23.

Holdsworth, D. W., \& Thornton, M. M. (2002). Micro-CT in small animal and specimen imaging. Trends in Biotechnology, 20(8), S34-S39.

Kazakia, G. J., Burghardt, A. J., Cheung, S., \& Majumdar, S. (2008). Assessment of bone tissue mineralization by conventional $\mathrm{x}$-ray microcomputed tomography: comparison with synchrotron radiation microcomputed tomography and ash measurements. Med Phys, 35(7), 3170-3179.

Kilkenny, C., Browne, W. J., Cuthill, I. C., Emerson, M., \& Altman, D. G. (2010). Improving bioscience research reporting: the ARRIVE guidelines for reporting animal research. PLOS Biol, 8(6), e1000412. doi:10.1371/journal.pbio.1000412

Lakin, B. A., Patel, H., Holland, C., Freedman, J. D., Shelofsky, J. S., Snyder, B. D., . . Grinstaff, M. W. (2016). Contrast-enhanced CT using a cationic contrast agent enables non-destructive assessment of the biochemical and biomechanical properties of mouse tibial plateau cartilage. Journal of Orthopaedic Research, 34(7), 1130-1138.

Laperre, K., Depypere, M., van Gastel, N., Torrekens, S., Moermans, K., Bogaerts, R., . . Carmeliet, G. (2011). Development of micro-CT protocols for in vivo follow-up of mouse bone architecture without major radiation side effects. Bone, 49(4), 613-622.

Li, H., Zhang, H., Tang, Z., \& Hu, G. (2008). Micro-computed tomography for small animal imaging: technological details. Progress in natural science, 18(5), 513-521.

Little, C. B., Barai, A., Burkhardt, D., Smith, S. M., Fosang, A. J., Werb, Z., et al. (2009). Matrix metalloproteinase 13-deficient mice are resistant to osteoarthritic cartilage erosion but not chondrocyte hypertrophy or osteophyte development. Arthritis and Rheumatism, 60(12), 3723-3733.

Little, C. B., \& Hunter, D. J. (2013). Post-traumatic osteoarthritis: from mouse models to clinical trials. Nature Reviews Rheumatology, 9(8), 485.

Lorenz, J., \& Grassel, S. (2014). Experimental osteoarthritis models in mice. Methods Mol Biol, 1194, 401-419.

Lusic, H., \& Grinstaff, M. W. (2013). X-ray-computed tomography contrast agents. Chem Rev, 113(3), 1641-1666.

Ma, H.-L., Blanchet, T. J., Peluso, D., Hopkins, B., Morris, E. A., \& Glasson, S. S. (2007). Osteoarthritis severity is sex dependent in a surgical mouse model. Osteoarthritis and Cartilage / OARS, Osteoarthritis Research Society, 15(6), 695-700.

McAlindon, T. E., Bannuru, R. R., Sullivan, M. C., Arden, N. K., Berenbaum, F., Bierma-Zeinstra, S. M., Hawker, G. A., Henrotin, Y., Hunter, D. J., Kawaguchi, H., Kwoh, K., Lohmander, S., Rannou, F., Roos, E. M., and Underwood, M (2014). OARSI guidelines for the non-surgical management of knee osteoarthritis. Osteoarthritis and Cartilage / OARS, Osteoarthritis Research Society, 22(3), 363-388.

Metscher, B. D. (2009). MicroCT for comparative morphology: simple staining methods allow highcontrast 3D imaging of diverse non-mineralized animal tissues. BMC Physiol, 9, 11.

Missbach-Guentner, J., Pinkert-Leetsch, D., Dullin, C., Ufartes, R., Hornung, D., Tampe, B., . . Alves, F. (2018). 3D virtual histology of murine kidneys-high resolution visualization of pathological alterations by micro computed tomography. Scientific Reports, 8(1), 1407.

Ondresik, M., Azevedo Maia, F. R., da Silva Morais, A., Gertrudes, A. C., Dias Bacelar, A. H., Correia, C., Goncalves, C., Radhouani, H. Amandi Sousa, R., Oliveira, J. M., \& Reis R. L. (2016). Management of Knee Osteoarthritis. Current Status and Future Trends. Biotech and Bioeng., 114, 717-739. 
Pauwels, E., Van Loo, D., Cornillie, P., Brabant, L., \& Van Hoorebeke, L. (2013). An exploratory study of contrast agents for soft tissue visualization by means of high resolution X-ray computed tomography imaging. J Microsc, 250(1), 21-31.

Postnov , A., Vinogradov , A., Van Dyck, D., Saveliev, S., \& De Clerck, N. (2003). Quantitative analysis of bone mineral content by $\mathrm{x}$-ray microtomography. Physiol. Meas., 24, 165-178.

Poulet, B., Hamilton, R. W., Shefelbine, S., \& Pitsillides, A. A. (2011). Characterizing a novel and adjustable noninvasive murine joint loading model. Arthritis \& Rheumatology, 63(1), 137147.

Raven, C. (1998). Numerical removal of ring artefacts in microtomography. Rev. Sci. Instrum., 69, 2978-2985.

Reginato A., Olsen B. (2002). The role of structural genes in the pathogenesis of osteoarthritic disorders. Arthritis Res, 4, 337-346.

Schambach, S. J., Bag, S., Schilling, L., Groden, C., \& Brockmann, M. A. (2010). Application of micro$\mathrm{CT}$ in small animal imaging. Methods, 50(1), 2-13.

Schneider, C. A., et al. (2012). NIH Image to ImageJ: 25 years of image analysis. Nature Methods, 9, 671-675.

Smith M., Little C. (2007). In: Moskowitz Rea, Ed. Experimental models of osteoarthritis. Philadelphia: Lippincott Williams \& Wilkins, 107e25.

Sorge, R. E., Martin, L. J., Isbester, K. A., Sotocinal, S. G., Rosen, S., Tuttle, A. H., . . Mogil, J. S. (2014). Olfactory exposure to males, including men, causes stress and related analgesia in rodents. Nat Methods, 11(6), 629-632. doi:10.1038/nmeth.2935

Staines K.A., Poulet B., Wentworth D.N., \& Pitsillides A.A. (2017). The STR/ort mouse model of spontaneous osteoarthritis - an update Osteoarthritis and Cartilage, 25 (6), 802-808.

Sutter, S., Todorov, A., Ismail, T., Haumer, A., Fulco, I., Schulz, G., . . Schaefer, D. J. (2017). ContrastEnhanced Microtomographic Characterisation of Vessels in Native Bone and Engineered Vascularised Grafts Using Ink-Gelatin Perfusion and Phosphotungstic Acid. Contrast media \& molecular imaging, 2017.

Vincent, T. L., Williams, R. O., Maciewicz, R., Silman, A., Garside, P., for the Arthritis Research UK animal models working group. (2012a). Mapping pathogenesis of arthritis through small animal models. Rheumatology (Oxford, England), 51(11), 1931-1941.

http://doi.org/10.1093/rheumatology/kes035

Vincent, T. L., Williams, R. O., Maciewicz, R., Silman, A., Garside, P., \& Group, A. R. U. A. M. W. (2012). Mapping pathogenesis of arthritis through small animal models. Rheumatology, 51(11), 1931-1941.

KEY REFERENCE (optional)

Glasson, S. S., Chambers, M. G., van den Berg, W. B., \& Little, C. B. (2010). The OARSI histopathology initiative - recommendations for histological assessments of osteoarthritis in the mouse. (Vol. 18, pp. S17-23). Presented at the Osteoarthritis and cartilage / OARS, Osteoarthritis Research Society.

INTERNET RESOURCES (optional)

\section{FIGURE LEGENDS}

Figure 1. DMM by cutting the meniscotibial ligament. 
After the surgical site has been shaved and cleaned with iodine a $1 \mathrm{~cm}$ incision is made lateral to the patella tendon through the skin and joint capsule. The medial meniscus is identified $(A)$ and the anterior attachment is identified and cut (B). Mobility of the meniscus is checked to ensure complete ligament transection. The joint capsule and skin are closed with sutures and the animal recovered. Further details can be found on Glasson, Blanchet, \& Morris, 2007).

Figure 2: Dartec HC10 loading machine used to apply axial loading to mouse knee joint. During the procedure, a mouse is anaesthetized using $4 \%$ isoflurane and hind limb is positioned between customdesigned upper and lower loading cups ensuring that the right ankle is securely placed in the lower cup and that the tibia is vertical.

Figure 3: Mouse knee joint

Dissected mouse knee joint showing the lateral muscle, patella tendon, tibia and femur.

Figure 4: Coronal embedding of the mouse knee

The knee joints are embedded coronally with the patella tendon $(A)$ positioned at the bottom of the metallic mould along its long axis (B).

F: Femur, PTd: patella tendon, T: tibia.

Figure 5: Paraffin block orientation in the microtome

The paraffin block is placed in the microtome block holder parallel to the blade.

Figure 6: Folding of articular cartilage

Folding of the articular cartilage in the femur condyle (pointed by the arrow in A) and tibial plateau (pointed by the arrow in B) may occur during sectioning.

\section{Figure 7: Knee joint orientation}

The correct coronal orientation shows the menisci appearing simultaneously from the medial and lateral compartment of the joint. On the other hand, in the mouse joint orientated incorrectly, the articular cartilage in the lateral compartment is appearing earlier than the medial side.

F: femur, T: tibia, M: meniscus.

Figure 8: Mouse knees joint stained with Safranin $O$ and Fast green

A. $4 x$ magnification image of the medial and lateral compartment of the knee joint.

10x magnification images of the medial (B) and lateral (C) compartments are acquired for scoring of cartilage damage. M: meniscus.

Figure 9. Mouse knee joint imaging by micro-CT.

A) Anterior, medial, and posterior views of a 3D rendering showing a mouse knee joint imaged by micro-CT ( $50 \mathrm{kV}, 200 \mu \mathrm{A}, 5 \mu \mathrm{m} / \mathrm{pixel}$ ). B) Representative slices of middle coronal, medial sagittal and cross-sectional views highlight the features observed in cortical and trabecular bone (L=lateral, $\mathrm{M}=$ medial, $\mathrm{A}=$ anterior, $\mathrm{P}=$ posterior).

Figure 10. Calibration of BMD. 
A) Projection image showing the calibration rods throughout scanning; note that the difference in density is visually distinctive. The dashed yellow lines delimit suitable volumes for analysis. Representative cross-sectional views of reconstructed B) $0.75 \mathrm{~g} / \mathrm{cm}^{3}$ and C) $0.25 \mathrm{~g} / \mathrm{cm}^{3}$ calcium hydroxyapatite phantom rods and correspondent histograms of grey level intensity obtained in the regions-of-interest marked in yellow. The linear relationship between the mineral

Figure 11.

Contrast-enhanced micro-CT imaging of mouse knee joints using PTA. Representative sagittal views of knee joints ( $50 \mathrm{kV}, 200 \mu \mathrm{A}, 5 \mu \mathrm{m} /$ pixel, 24 hours of incubation) showing: A) incomplete staining, thus creating 'gaps' in the articular cartilage (highlighted by red arrows) and B) overlap among articular surfaces of femur, menisci and tibia.

Figure 12: Linton incapacitance tester

The incapacitance tester $(A)$ and the correct placement of of the animal in the testing chamber. The animal is symmetrical and still with the tail lightly held in place (B).

Figure 13. Effect of spatial resolution on $\mu C T$ imaging. Representative coronal views of a mouse knee joint imaged at A) $5 \mu \mathrm{m} /$ pixel $(50 \mathrm{kV}, 200 \mu \mathrm{A})$ and B) $10 \mu \mathrm{m} /$ pixel $(70 \mathrm{kV}, 160 \mu \mathrm{A})$, in which blurring of trabecular bone is perceptible, decreasing image quality.

Figure 14. Contrast-enhanced micro-CT imaging of mouse articular cartilage using PTA applied to a surgical mouse model of knee osteoarthritis (destabilisation of the medial meniscus, DMM). Representative coronal views of pairs of naïve, 4- and 12-week post-DMM tibiae (50 kV, $200 \mu \mathrm{A}$, $5 \mu \mathrm{m} / \mathrm{pixel}, 24$ hours of incubation); zoomed views of the medial compartment in DMM-operated enhance the presence of lesions (indicated by red arrows) due to pathology.

Figure 15: Scoring system

Semi-quantitative scoring system modified from Glasson et al. $^{3}$

A. Representative images of stained cartilage with corresponding scores. B. More detailed description of the scoring system.

Figure 16: An example of histological analysis of DMM induced disease in mice.

The graph represents the summed histologic scores of knee cartilage from C57BL/6J mice at 4, 8, 12 and 20 weeks after DMM surgery.

TABLES

\begin{tabular}{|c|c|c|}
\hline \multicolumn{3}{|c|}{ Use of volatile agents in rodents and rabbits } \\
\hline \multirow[t]{2}{*}{ Anaesthetic } & \multicolumn{2}{|c|}{ Concentration (\%) } \\
\hline & Induction & Maintenance \\
\hline Halothane & $2.0-4.0$ & $0.8-2.0$ \\
\hline \multicolumn{3}{|l|}{ Isoflurane } \\
\hline Small animals & $2.0-3.0$ & $0.25-2.0$ \\
\hline
\end{tabular}




\begin{tabular}{|c|l|l|}
\hline Large animals & $3.0-4.0$ & $1.5-3.0$ \\
\hline
\end{tabular}

Table 1. Guide concentrations on inhaled anasthaesia in rodents (From 'Handbook of laboratory animal management and welfare' Wolfensohn and Lloyd)

Video 1: Coronal embedding of the mouse knee 